\title{
Corrigendum: Micro- and Nanotechnologies for Optical Neural Interfaces
}

\author{
Ferruccio Pisanello ${ }^{1 *}$, Leonardo Sileo ${ }^{1}$ and Massimo De Vittorio ${ }^{1,2 *}$ \\ ${ }^{1}$ Center for Biomolecular Nanotechnologies, Istituto Italiano di Tecnologia, Lecce, Italy, ${ }^{2}$ Dipartimento di Ingegneria \\ dell'Innovazione, Università del Salento, Lecce, Italy
}

Keywords: nanotechnology, optogenetics, nanoparticles, neural interfaces, optical fibers

\section{A corrigendum on}

\section{OPEN ACCESS}

Edited and reviewed by: Ruxandra Vidu,

University of California, Davis, USA

${ }^{*}$ Correspondence:

Ferruccio Pisanello ferruccio.pisanello@iit.it

Massimo De Vittorio

massimo.devittorio@iit.it

Specialty section

This article was submitted to

Neural Technology,

a section of the journal

Frontiers in Neuroscience

Received: 05 September 2016

Accepted: 29 September 2016

Published: 14 October 2016

Citation:

Pisanello F, Sileo $L$ and De Vittorio $M$

(2016) Corrigendum: Micro- and Nanotechnologies for Optical Neural Interfaces. Front. Neurosci. 10:468.

doi: 10.3389/fnins.2016.00468
Micro- and Nanotechnologies for Optical Neural Interfaces

by Pisanello, F., Sileo, L., and De Vittorio, M. (2016). Front. Neurosci. 10:70. doi: 10.3389/fnins.2016.00070

After the publication of this paper Pisanello et al., we have noticed that there was an error in the institutional affiliations of some of the authors. In particular, Leonardo Sileo, who appeared affiliated to "Dipartimento di Ingegneria dell'Innovazione, Università del Salento, Lecce, Italy", should be affiliated instead only to "Center for Biomolecular Nanotechnologies, Istituto Italiano di Tecnologia, Lecce, Italy." Massimo De Vittorio, who appeared affiliated only to "Center for Biomolecular Nanotechnologies, Istituto Italiano di Tecnologia, Lecce, Italy" should be affiliated also to "Dipartimento di Ingegneria dell'Innovazione, Università del Salento, Lecce, Italy." Correct affiliations appear below the title of this corrigendum.

\section{AUTHOR CONTRIBUTIONS}

All authors contributed equally to this work.

Conflict of Interest Statement: The authors declare that the research was conducted in the absence of any commercial or financial relationships that could be construed as a potential conflict of interest.

Copyright $\odot 2016$ Pisanello, Sileo and De Vittorio. This is an open-access article distributed under the terms of the Creative Commons Attribution License (CC BY). The use, distribution or reproduction in other forums is permitted, provided the original author(s) or licensor are credited and that the original publication in this journal is cited, in accordance with accepted academic practice. No use, distribution or reproduction is permitted which does not comply with these terms. 